\title{
Intramolecular Benzyl Protection Delivery: A Practical Synthesis of DMDP and DGDP from D-Fructose
}

\author{
M. Isabel García-Moreno, ${ }^{\dagger}$ Matilde Aguilar, ${ }^{\dagger}$ Carmen Ortiz Mellet, ${ }^{*}, \dagger$ José M. García \\ Fernández ${ }^{*}$;
}

\section{Supporting Information}

General Methods. Reagents and solvents were purchased from commercial sources and used without further purification, with the following exceptions: DMF was distilled form $\mathrm{BaO}$, dichloromethane was distilled under $\mathrm{Ar}$ stream over $\mathrm{CaH}_{2}$, toluene was distilled under Ar stream over sodium, and pyridine was distilled from KOH. Optical rotations were measured at $20{ }^{\circ} \mathrm{C}$ in $1-\mathrm{cm}$ or $1-\mathrm{dm}$ tubes on a Perkin-Elmer $141 \mathrm{MC}$ polarimeter. IR spectra were recorded on a Bomem Michelson MB-120 FTIR spectrometer. ${ }^{1} \mathrm{H}$ (and ${ }^{13} \mathrm{C}$ NMR) spectra were recorded at 500 (125.7) and 300 (75.5) MHz with, respectively, Bruker 500 DRX spectrometers and 300 AMX spectrometers. 2D COSY and HMQC experiments were used to assist on NMR assignments. Thin-layer chromatography (TLC) was carried out on aluminium sheets coated with Kieselgel 30 F245 (E. Merck), with visualization by UV light and by charring with $10 \% \mathrm{H}_{2} \mathrm{SO}_{4}$. Column chromatography was carried out on Silica Gel 60 (E. Merck, 230-400 mesh). FAB mass spectra were obtained with a Kratos MS-80 RFA instrument. The operating conditions were the following: the primary beam consisted of Xe atoms with a maximum energy of $8 \mathrm{keV}$; the samples were dissolved in thioglycerol, and the positive ions were separated and accelerated over a potential of $7 \mathrm{keV}$; $\mathrm{NaI}$ was added as cationizing agent. Elemental analyses were performed at the Instituto de Investigaciones Químicas (Sevilla, Spain).

\section{3-O-(2-Bromomethyl)benzyl-1,2:4,5-di- $O$-isopropylidene- $\beta$-D-fructopyranose}

(6). To a solution of commercial 1,2-dibromomethylbencene $(5.97 \mathrm{~g}, 22.6 \mathrm{mmol})$ in dry DMF $(150 \mathrm{~mL}), \mathrm{NaH}(60 \%$ in mineral oil, $1.14 \mathrm{~g}, 28.35 \mathrm{mmol})$ was added and the suspension was stirred at room temperature for $20 \mathrm{~min}$. 1,2:4,5-Di- $O$-isopropylidene- $\beta$-D- 
fructopyranose $5(3.0 \mathrm{~g}, 11.34 \mathrm{mmol})$ was then added and the reaction mixture was further stirred for $24 \mathrm{~h} . \mathrm{Et}_{2} \mathrm{O}(50 \mathrm{~mL})$ and water $(50 \mathrm{~mL})$ were added, the organic layer was separated, washed with water $(5 \times 25 \mathrm{~mL})$, dried $\left(\mathrm{MgSO}_{4}\right)$, filtered, and concentrated. The resulting residue was purified by column chromatography (1:8 EtOAc-petroleum ether) to yield $6(2.34 \mathrm{~g}, 91 \%) ; R_{f}=0.73\left(2: 5\right.$ EtOAc-petroleum ether); $[\alpha]_{\mathrm{D}}=-38.6\left(c 0.8, \mathrm{CHCl}_{3}\right)$; ${ }^{1} \mathrm{H}$ NMR (500 MHz, $\left.\mathrm{CDCl}_{3}\right): \delta=7.28-7.24(\mathrm{~m}, 4 \mathrm{H}, \mathrm{Ph}), 5.08,4.76\left(2 \mathrm{~d}, 2 \mathrm{H},{ }^{2} \mathrm{~J}_{\mathrm{H}, \mathrm{H}}=12.0\right.$ $\mathrm{Hz}, \mathrm{CHPh}), 4.73,4.58\left(2 \mathrm{~d}, 2 \mathrm{H},{ }^{2} J_{\mathrm{H}, \mathrm{H}}=10.2 \mathrm{~Hz}, \mathrm{CHBr}\right), 4.36\left(\mathrm{dd}, 1 \mathrm{H}, J_{3,4}=7.2 \mathrm{~Hz}, J_{4,5}=\right.$ $5.6 \mathrm{~Hz}, \mathrm{H}-4), 4.21$ (dd, $\left.1 \mathrm{H}, J_{5,6 \mathrm{a}}=2.5 \mathrm{~Hz}, \mathrm{H}-5\right), 3.96$ (dd, 1 H, $\left.J_{6 \mathrm{a}, 6 \mathrm{~b}}=13.4 \mathrm{~Hz}, \mathrm{H}-6 \mathrm{a}\right), 3.99$ (d, $1 \mathrm{H}, \mathrm{H}-6 \mathrm{~b}), 3.96$ (d, $\left.1 \mathrm{H}, J_{1 \mathrm{a}, 1 \mathrm{~b}}=8.8 \mathrm{~Hz}, \mathrm{H}-1 \mathrm{a}\right), 3.88$ (d, $\left.1 \mathrm{H}, \mathrm{H}-1 \mathrm{~b}\right), 3.53$ (d, $1 \mathrm{H}, \mathrm{H}-3$ ), 1.60, 1.55, 1.37, $1.36\left(4 \mathrm{~s}, 12 \mathrm{H}, \mathrm{CMe}_{2}\right) ;{ }^{13} \mathrm{C} \mathrm{NMR}\left(125.7 \mathrm{MHz}, \mathrm{CDCl}_{3}\right): \delta=136.8-128.5$ (Ph), 112.1, $109.1\left(\mathrm{CMe}_{2}\right), 104.3$ (C-2), 77.5 (C-4), 76.8 (C-3), 73.8 (C-5), 72.2 (C-1), 70.8 $\left(\mathrm{CH}_{2} \mathrm{Ph}\right), 60.4$ (C-6), $31.1\left(\mathrm{CH}_{2} \mathrm{Br}\right), 28.2,26.6,26.3,26.2\left(\mathrm{CMe}_{2}\right)$; FABMS: $m / z$ 465, 467 $\left(80 \%,[\mathrm{M}+\mathrm{Na}]^{+}\right)$. Anal. Calcd for $\mathrm{C}_{20} \mathrm{H}_{27} \mathrm{BrO}_{6}: \mathrm{C}, 54.18 ; \mathrm{H}, 6.14$. Found: $\mathrm{C}, 54.08 ; \mathrm{H}, 6.30$.

\section{3-O-(2-Bromomethyl)benzyl-1,2- $O$-isopropylidene- $\beta$-D-fructopyranose}

Compound $6(1.88 \mathrm{~g}, 4.23 \mathrm{mmol})$ was dissolved in $60 \%$ aq AcOH $(15 \mathrm{~mL})$ and stirred at 45 ${ }^{\circ} \mathrm{C}$ for $2 \mathrm{~h}$ The reaction mixture was then diluted with water $(10 \mathrm{~mL})$ and extracted with EtOAc $(4 \times 10 \mathrm{~mL})$. The combined organic phase was washed with saturated aq $\mathrm{NaHCO}_{3}$ $(10 \mathrm{~mL})$, dried $\left(\mathrm{MgSO}_{4}\right)$, filtered, and concentrated. The resulting residue was purified by column chromatography $(2: 3 \rightarrow 1: 1$ EtOAc-petroleum ether $)$ to give $7(1.5 \mathrm{~g}, 87 \%) ; R_{f}=$ 0.47 (3:1 EtOAc-petroleum ether); $[\alpha]_{\mathrm{D}}=-60.3\left(c 0.8\right.$ in $\left.\mathrm{CHCl}_{3}\right) ;{ }^{1} \mathrm{H}$ NMR $(500 \mathrm{MHz}$, $\left.\mathrm{CD}_{3} \mathrm{OD}\right): \delta=7.37-7.26(\mathrm{~m}, 4 \mathrm{H}, \mathrm{Ph}), 5.15,4.75\left(2 \mathrm{~d}, 2 \mathrm{H},{ }^{2} J_{\mathrm{H}, \mathrm{H}}=11.6 \mathrm{~Hz}, \mathrm{CHPh}\right), 4.90$, $4.66\left(2 \mathrm{~d}, 2 \mathrm{H},{ }^{2} J_{\mathrm{H}, \mathrm{H}}=10.2 \mathrm{~Hz}, \mathrm{CHBr}\right), 3.93\left(\mathrm{dd}, 1 \mathrm{H}, J_{3,4}=9.8 \mathrm{~Hz}, J_{4,5}=3.5 \mathrm{~Hz}, \mathrm{H}-4\right)$, $3.89\left(\mathrm{dd}, 1 \mathrm{H}, J_{6 \mathrm{a}, 6 \mathrm{~b}}=12.4 \mathrm{~Hz}, J_{5,6 \mathrm{a}}=1.4 \mathrm{~Hz}, \mathrm{H}-6 \mathrm{a}\right), 3.85\left(\mathrm{~d}, 1 \mathrm{H}, J_{1 \mathrm{a}, 1 \mathrm{~b}}=8.6 \mathrm{~Hz}, \mathrm{H}-1 \mathrm{a}\right)$, 3.84 (m, 1 H, H-5), 3.80 (d, 1 H, H-1b), 3.69 (d, 1 H, H-3), 3.62 (d, 1 H, J5,6b $=1.9$ Hz, H6b), 1.39, $1.30\left(2 \mathrm{~s}, 6 \mathrm{H}, \mathrm{CMe}_{2}\right) ;{ }^{13} \mathrm{C} \mathrm{NMR}\left(125.7 \mathrm{MHz}, \mathrm{CD}_{3} \mathrm{OD}\right): \delta=137.1-128.1(\mathrm{Ph})$, $111.5\left(\mathrm{CMe}_{2}\right), 105.8(\mathrm{C}-2), 75.8(\mathrm{C}-3), 71.8\left(\mathrm{CH}_{2} \mathrm{Ph}\right), 71.7$ (C-1), 71.5 (C-4), 70.1 (C-5), $64.3(\mathrm{C}-6), 30.5\left(\mathrm{CH}_{2} \mathrm{Br}\right), 25.7,25.2\left(\mathrm{CMe}_{2}\right)$; FABMS: $m / z$ 425, $427\left(70 \%,[\mathrm{M}+\mathrm{Na}]^{+}\right)$. Anal. Calcd for $\mathrm{C}_{17} \mathrm{H}_{23} \mathrm{BrO}_{6}$ : C 50.63, H 5.75. Found: C, 50.23; H, 5.79.

1,2-O-Isopropylidene-3,4-O-(o-xylylene)- $\beta$-D-fructopyranose (8). To a solution of 7 (470 mg, $1.16 \mathrm{mmol}$ ) in DMF (3 mL), NaH (60\% in mineral oil, $58 \mathrm{mg}, 1.4 \mathrm{mmol}, 2.5$ eq) was added and the suspension was stirred at room temperature for $15 \mathrm{~min}$. $\mathrm{Et}_{2} \mathrm{O}$ (12 
$\mathrm{mL})$ and $\mathrm{H}_{2} \mathrm{O}(8 \mathrm{~mL})$ were then added, the organic phase was decanted, dried $\left(\mathrm{MgSO}_{4}\right)$, filtered, and concentrated. The resulting residue was purified by column chromatography (1:2 EtOAc-petroleum ether) to yield 8 (348 mg, 93\%); $R_{f}=0.65$ (1:1 EtOAc-petroleum ether); $[\alpha]_{\mathrm{D}}=-189.5\left(c 0.8, \mathrm{CHCl}_{3}\right) ;{ }^{1} \mathrm{H}$ NMR (500 MHz, $\left.\mathrm{CDCl}_{3}\right): \delta=7.25-7.05(\mathrm{~m}, 4 \mathrm{H}$, $\mathrm{Ph}), 5.27,4.76\left(2 \mathrm{~d}, 2 \mathrm{H},{ }^{2} J_{\mathrm{H}, \mathrm{H}}=12.9 \mathrm{~Hz}, \mathrm{CHPh}\right), 5.18,4.81\left(2 \mathrm{~d}, 2 \mathrm{H},{ }^{2} J_{\mathrm{H}, \mathrm{H}}=14.9 \mathrm{~Hz}\right.$, $\mathrm{CHPh}), 4.09$ (d, $\left.1 \mathrm{H}, J_{1 \mathrm{a}, 1 \mathrm{~b}}=8.5 \mathrm{~Hz}, \mathrm{H}-1 \mathrm{a}\right), 4.01$ (d, $\left.1 \mathrm{H}, \mathrm{H}-1 \mathrm{~b}\right), 3.99$ (m, $\left.1 \mathrm{H}, \mathrm{H}-5\right), 3.91$ (d, $\left.1 \mathrm{H}, J_{6 \mathrm{a}, 6 \mathrm{~b}}=12.7 \mathrm{~Hz}, \mathrm{H}-6 \mathrm{a}\right), 3.87\left(\mathrm{dd}, 1 \mathrm{H}, J_{3,4}=9.4 \mathrm{~Hz}, J_{4,5}=3.5 \mathrm{~Hz}, \mathrm{H}-4\right), 3.74$ (d, $1 \mathrm{H}$, H-3), 3.73 (dd, $\left.1 \mathrm{H}, J_{5,6 \mathrm{~b}}=1.6 \mathrm{~Hz}, \mathrm{H}-6 \mathrm{~b}\right), 1.48,1.42\left(2 \mathrm{~s}, 6 \mathrm{H}, \mathrm{CMe}_{2}\right) ;{ }^{13} \mathrm{C} \mathrm{NMR}(125.7$ $\left.\mathrm{MHz}, \mathrm{CDCl}_{3}\right): \delta=136.8-127.8(\mathrm{Ph}), 112.0\left(\mathrm{CMe}_{2}\right), 105.6(\mathrm{C}-2), 77.1$ (C-4), 75.7 (C-3), 73.7, $72.0\left(\mathrm{CH}_{2} \mathrm{Ph}\right), 71.6(\mathrm{C}-1), 68.8$ (C-5), 63.1 (C-6), 27.1, $26.1\left(\mathrm{CMe}_{2}\right)$; FABMS: $\mathrm{m} / \mathrm{z}$ $345\left(95 \%,[\mathrm{M}+\mathrm{Na}]^{+}\right)$. Anal. Calcd for $\mathrm{C}_{17} \mathrm{H}_{22} \mathrm{O}_{6}$ : C 63.34, H 6.88. Found: C 63.27, H 6.99.

5-Deoxy-5-iodo-1,2-O-isopropylidene-3,4-O-(o-xylylene)- $\alpha$-L-sorbopyranose (9). To a solution of 8 (764 mg, $2.36 \mathrm{mmol}), \mathrm{PPh}_{3}(2.04 \mathrm{~g}, 7.78 \mathrm{mmol})$ and imidazole $(530 \mathrm{mg}$, $7.78 \mathrm{mmol})$ in dry toluene $(52 \mathrm{~mL})$, iodine $(1.32 \mathrm{~g}, 5.2 \mathrm{mmol})$ was added. The reaction mixture was stirred at $80{ }^{\circ} \mathrm{C}$ for $16 \mathrm{~h}$, then cooled, an equal volume of saturated aq $\mathrm{NaHCO}_{3}$ was added and the mixture was stirred for 5 min. Iodine was added in portions until the toluene phase remained iodine-colored and the mixture was additionally stirred for $10 \mathrm{~min}$. Excess of iodine was removed by addition of $10 \%(\mathrm{w} / \mathrm{v})$ aq $\mathrm{Na}_{2} \mathrm{~S}_{2} \mathrm{O}_{3}$, the organic phase was separated, washed with water, dried $\left(\mathrm{MgSO}_{4}\right)$, filtered, and concentrated. The resulting residue was purified by column chromatography $(1: 6 \rightarrow 1: 4$ EtOAc-petroleum ether) to yield $9(1.004 \mathrm{~g}, 98 \%) ; R_{f}=0.57\left(1: 4\right.$ EtOAc-petroleum ether); $[\alpha]_{\mathrm{D}}=-43.6(c$ 1.0, $\mathrm{CH}_{2} \mathrm{Cl}_{2}$ ); IR (KBr): $v_{\max }=3030,2950,1643,1452,1389,1095,713 \mathrm{~cm}^{-1} ;{ }^{1} \mathrm{H} \mathrm{NMR}$ $\left(500 \mathrm{MHz}, \mathrm{CDCl}_{3}\right): \delta=7.23-7.10(\mathrm{~m}, 4 \mathrm{H}, \mathrm{Ph}), 5.16,5.07\left(2 \mathrm{~d}, 2 \mathrm{H},{ }^{2} J_{\mathrm{H}, \mathrm{H}}=14.5 \mathrm{~Hz}\right.$, $\mathrm{CHPh}), 5.00,4.97\left(2 \mathrm{~d}, 2 \mathrm{H},{ }^{2} J_{\mathrm{H}, \mathrm{H}}=14.5 \mathrm{~Hz}, \mathrm{CHPh}\right), 4.16\left(\mathrm{~d}, 1 \mathrm{H}, J_{1 \mathrm{a}, 1 \mathrm{~b}}=8.5 \mathrm{~Hz}, \mathrm{H}-1 \mathrm{a}\right)$, $4.03\left(\mathrm{t}, 1 \mathrm{H}, J_{6 \mathrm{a}, 6 \mathrm{~b}}=J_{5,6 \mathrm{a}}=11.0 \mathrm{~Hz}, \mathrm{H}-6 \mathrm{a}\right), 3.93$ (d, $\left.1 \mathrm{H}, \mathrm{H}-1 \mathrm{~b}\right), 3.87$ (m, $\left.2 \mathrm{H}, \mathrm{H}-5, \mathrm{H}-4\right)$, $3.80\left(\mathrm{dd}, 1 \mathrm{H}, J_{5,6 \mathrm{~b}}=5.0 \mathrm{~Hz}, \mathrm{H}-6 \mathrm{~b}\right), 3.37\left(\mathrm{~d}, 1 \mathrm{H}, J_{3,4}=9.0 \mathrm{~Hz}, \mathrm{H}-3\right), 1.46,1.37(2 \mathrm{~s}, 6 \mathrm{H}$, $\left.\mathrm{CMe}_{2}\right) ;{ }^{13} \mathrm{C}$ NMR $\left(125.7 \mathrm{MHz}, \mathrm{CDCl}_{3}\right): \delta=136.9-127.8(\mathrm{Ph}), 112.6\left(\mathrm{CMe}_{2}\right), 105.4(\mathrm{C}-2)$, 83.6 (C-4), 80.4 (C-3), 74.2, $73.5\left(\mathrm{CH}_{2} \mathrm{Ph}\right), 71.8$ (C-1), 65.7 (C-6), 27.1, $25.9\left(\mathrm{CMe}_{2}\right), 26.3$ (C-5); FABMS: $m / z 455\left(50 \%,[\mathrm{M}+\mathrm{Na}]^{+}\right)$. Anal. Calcd for $\mathrm{C}_{17} \mathrm{H}_{21} \mathrm{IO}_{5}$ : C 47.24, H 4.90. Found: C, 47.34; H, 4.90. 


\section{5-Azido-5-deoxy-1,2- $O$-isopropylidene-3,4- $O$-( $O$-xylylene)- $\beta$-D-fructopyranose}

(10). To a solution of 9 (469 mg, $1.08 \mathrm{mmol})$ in HMPT (4.5 mL), $\mathrm{NaN}_{3}$ (353 mg, 5.43 mmol) was added and the resulting suspension was stirred at $80^{\circ} \mathrm{C}$ for $12 \mathrm{~h}$. The reaction mixture was cooled, EtOAc $(15 \mathrm{~mL})$ and $\mathrm{H}_{2} \mathrm{O}(15 \mathrm{~mL})$ were added, the organic phase was decanted and the aqueous phase was extracted with EtOAc $(5 \times 10 \mathrm{~mL})$. The combined organic extracts were washed with water $(2 \times 10 \mathrm{~mL})$, dried $\left(\mathrm{MgSO}_{4}\right)$, filtered and concentrated. The resulting solid residue was purified by column chromatography (1:8 EtOAc-petroleum ether) to yield 10 (342 mg, 91\%); $R_{f} 0.44$ (1:4 EtOAc-petroleum ether); $[\alpha]_{\mathrm{D}}=-181.8\left(c\right.$ 0.99, $\left.\mathrm{CH}_{2} \mathrm{Cl}_{2}\right)$; IR (KBr): $v_{\max }=3030,2984,2110,1650,1451,1380$, 1094, $768 \mathrm{~cm}^{-1} ;{ }^{1} \mathrm{H}$ NMR (400 MHz, $\left.\mathrm{CDCl}_{3}\right): \delta=7.27-7.07$ (m, $\left.4 \mathrm{H}, \mathrm{Ph}\right), 5.17,4.89$ (2 d, 2 $\left.\mathrm{H},{ }^{2} J_{\mathrm{H}, \mathrm{H}}=12.0 \mathrm{~Hz}, \mathrm{CHPh}\right), 5.15,4.86\left(2 \mathrm{~d}, 2 \mathrm{H},{ }^{2} J_{\mathrm{H}, \mathrm{H}}=10.0 \mathrm{~Hz}, \mathrm{CHPh}\right), 4.12\left(\mathrm{~d}, 1 \mathrm{H}, J_{\mathrm{la}, 1 \mathrm{~b}}\right.$ $=8.4 \mathrm{~Hz}, \mathrm{H}-1 \mathrm{a}), 4.04\left(\mathrm{dd}, 1 \mathrm{H}, J_{3,4}=9.6 \mathrm{~Hz}, J_{4,5}=4.0 \mathrm{~Hz}, \mathrm{H}-4\right), 3.99$ (d, $\left.1 \mathrm{H}, \mathrm{H}-1 \mathrm{~b}\right), 3.94$ $\left(\mathrm{dd}, 1 \mathrm{H}, J_{6 \mathrm{a}, 6 \mathrm{~b}}=12.8 \mathrm{~Hz}, J_{5,6 \mathrm{a}}=1.6 \mathrm{~Hz}, \mathrm{H}-6 \mathrm{a}\right), 3.93$ (m, $\left.1 \mathrm{H}, \mathrm{H}-5\right), 3.75$ (d, $\left.1 \mathrm{H}, \mathrm{H}-3\right), 3.64$ $\left(\mathrm{dd}, 1 \mathrm{H}, J_{5,6 \mathrm{~b}}=2.0 \mathrm{~Hz}, \mathrm{H}-6 \mathrm{~b}\right), 1.46,1.40\left(2 \mathrm{~s}, 6 \mathrm{H}, \mathrm{CMe}_{2}\right) ;{ }^{13} \mathrm{C} \mathrm{NMR}\left(100.6 \mathrm{MHz}, \mathrm{CDCl}_{3}\right)$ : $\delta=136.5-127.9(\mathrm{Ph}), 112.2\left(\mathrm{CMe}_{2}\right), 105.5(\mathrm{C}-2), 78.1$ (C-4), 76.1 (C-3), 73.9, 72.4 $\left(\mathrm{CH}_{2} \mathrm{Ph}\right), 71.6$ (C-1), 61.9 (C-6), 61.1 (C-5), 27.0, $25.9\left(\mathrm{CMe}_{2}\right)$; FABMS: $m / z 370$ (50\%, $\left.[\mathrm{M}+\mathrm{Na}]^{+}\right), 332\left(25 \%,[\mathrm{M}+\mathrm{Me}]^{+}\right)$. Anal. Calcd for $\mathrm{C}_{17} \mathrm{H}_{21} \mathrm{~N}_{3} \mathrm{O}_{5}: \mathrm{C}, 58.78 ; \mathrm{H}, 6.09 ; \mathrm{N}, 12.09$. Found: C, 58.50; H, 5.85; N, 12.00 .

5-Azido-5-deoxy-3,4-O-(o-xylylene)- $\beta$-D-fructopyranose (11). A solution of 10 (279 $\mathrm{mg}, 0.80 \mathrm{mmol})$ in $90 \%$ aq TFA $(2.3 \mathrm{~mL})$ was stirred at room temperature for $30 \mathrm{~min}$. The mixture was concentrated and repeatedly co-distilled with water. The residue was purified by column chromatography (2:1 EtOAc-petroleum ether) to yield 11 (241 mg, 98\%); $R_{f} 0.33$ (3:1 EtOAc-petroleum ether); $[\alpha]_{\mathrm{D}}=-156.9\left(\right.$ c 1.0, $\left.\mathrm{CH}_{2} \mathrm{Cl}_{2}\right)$; IR $(\mathrm{KBr}): v_{\max }$ $=3032,2975,2104,1651,1449,1379,1094,748 \mathrm{~cm}^{-1} ;{ }^{1} \mathrm{H}$ NMR $\left(400 \mathrm{MHz}, \mathrm{CDCl}_{3}\right): \delta=$ 7.27-7.10 (m, $4 \mathrm{H}, \mathrm{Ph}), 5.12,4.91\left(2 \mathrm{~d}, 2 \mathrm{H},{ }^{2} J_{\mathrm{H}, \mathrm{H}}=14.0 \mathrm{~Hz}, \mathrm{CHPh}\right), 5.06,4.99(2 \mathrm{~d}, 2 \mathrm{H}$, $\left.{ }^{2} J_{\mathrm{H}, \mathrm{H}}=14.5 \mathrm{~Hz}, \mathrm{CHPh}\right), 4.05\left(\mathrm{dd}, 1 \mathrm{H}, J_{3,4}=9.0 \mathrm{~Hz}, J_{4,5}=3.5 \mathrm{~Hz}, \mathrm{H}-4\right), 3.99$ (dd, $1 \mathrm{H}, J_{6 \mathrm{a}, 6 \mathrm{~b}}$ $\left.=12.5 \mathrm{~Hz}, J_{5,6 \mathrm{a}}=1.0 \mathrm{~Hz}, \mathrm{H}-6 \mathrm{a}\right), 3.93(\mathrm{~m}, 1 \mathrm{H}, \mathrm{H}-5), 3.82$ (d, $\left.1 \mathrm{H}, \mathrm{H}-3\right), 3.65$ (m, $3 \mathrm{H}, \mathrm{H}-1 \mathrm{a}$, $\mathrm{H}-1 \mathrm{~b}, \mathrm{H}-6 \mathrm{~b}), 3.31,2.24$ (2 bs, $2 \mathrm{H}, \mathrm{OH}) ;{ }^{13} \mathrm{C} \mathrm{NMR}\left(100.6 \mathrm{MHz}, \mathrm{CDCl}_{3}\right): \delta=136.5-128.2$ (Ph), 97.7 (C-2), 77.4 (C-4), 76.0 (C-3), 73.5, $72.3\left(\mathrm{CH}_{2} \mathrm{Ph}\right), 65.7$ (C-1), 61.6 (C-6), 60.8 (C-5); FABMS: $m / z 330\left(30 \%,[\mathrm{M}+\mathrm{Na}]^{+}\right)$. Anal. Calcd for $\mathrm{C}_{14} \mathrm{H}_{17} \mathrm{~N}_{3} \mathrm{O}_{5}: \mathrm{C}, 54.72 ; \mathrm{H}, 5.58$; N, 13.67. Found: C, 54.75; H, 5.34; N, 13.52 . 


\section{5-Azido-5-deoxy-1,2- $O$-isopropylidene-3,4- $O$-(o-xylenylene)- $\alpha$-L-sorbopyranose}

(12). To a solution of $8(423 \mathrm{mg}, 1.31 \mathrm{mmol})$ in $\mathrm{CH}_{2} \mathrm{Cl}_{2}(8 \mathrm{~mL})$ at $-25{ }^{\circ} \mathrm{C}$, pyridine $(0.24$ $\mathrm{mL}, 2.88 \mathrm{mmol})$ and trifluoromethanesulfonic anhydride $(0.32 \mathrm{~mL}, 1.97 \mathrm{mmol})$ were added under $\mathrm{N}_{2}$. The reaction mixture was allowed to reach room temperature and stirred for $1 \mathrm{~h}$, then diluted with $\mathrm{CH}_{2} \mathrm{Cl}_{2}(10 \mathrm{~mL})$, washed with saturated aqueous $\mathrm{NaHCO}_{3}(8 \mathrm{~mL})$, dried $\left(\mathrm{MgSO}_{4}\right)$, and concentrated. The resulting crude trifluoromethanesulfonate ester was dissolved in DMF $(5.5 \mathrm{~mL}), \mathrm{NaN}_{3}(426 \mathrm{mg}, 6.55 \mathrm{mmol})$ was added and the reaction mixture was stirred at room temperature for $3 \mathrm{~h}$. $\mathrm{Et}_{2} \mathrm{O}(10 \mathrm{~mL})$ and $\mathrm{H}_{2} \mathrm{O}(10 \mathrm{~mL})$ were added, the organic phase was decanted and the aqueous phase was extracted with $\mathrm{Et}_{2} \mathrm{O}(5 \times$ $10 \mathrm{~mL})$. The organic extracts were washed with water $(2 \times 10 \mathrm{~mL})$, dried $\left(\mathrm{MgSO}_{4}\right)$, filtered, and concentrated. The resulting solid which was purified by column chromatography (1:8 EtOAc-petroleum ether) to yield 12 (378 mg, 83\%); $R_{f} 0.59$ (1:4 EtOAc-petroleum ether); $[\alpha]_{\mathrm{D}}=-211\left(c\right.$ 1.0, $\left.\mathrm{CH}_{2} \mathrm{Cl}_{2}\right)$; IR (KBr): $v_{\max }=3029,2979,2108,1645,1454,1377,1096$, $762 \mathrm{~cm}^{-1}$; ${ }^{1} \mathrm{H}$ NMR (500 MHz, $\left.\mathrm{CDCl}_{3}\right): \delta=7.27-7.09$ (m, $\left.4 \mathrm{H}, \mathrm{Ph}\right), 5.14,5.00(2 \mathrm{~d}, 2 \mathrm{H}$, $\left.{ }^{2} J_{\mathrm{H}, \mathrm{H}}=14.0 \mathrm{~Hz}, \mathrm{CHPh}\right), 5.08,4.92\left(2 \mathrm{~d}, 2 \mathrm{H},{ }^{2} J_{\mathrm{H}, \mathrm{H}}=14.5 \mathrm{~Hz}, \mathrm{CHPh}\right), 4.13\left(\mathrm{~d}, 1 \mathrm{H}, J_{1 \mathrm{a}, \mathrm{b}}=\right.$ $8.5 \mathrm{~Hz}, \mathrm{H}-1 \mathrm{a}), 3.93$ (d, $1 \mathrm{H}, \mathrm{H}-1 \mathrm{~b}), 3.79$ (t, $\left.1 \mathrm{H}, J_{3,4}=J_{4,5}=9.0 \mathrm{~Hz}, \mathrm{H}-4\right), 3.65$ (dd, $1 \mathrm{H}$, $\left.J_{6 \mathrm{a}, 6 \mathrm{~b}}=11.0 \mathrm{~Hz}, J_{5,6 \mathrm{a}}=5.0 \mathrm{~Hz}, \mathrm{H}-6 \mathrm{a}\right), 3.55\left(\mathrm{t}, 1 \mathrm{H}, J_{5,6 \mathrm{~b}}=11.0 \mathrm{~Hz}, \mathrm{H}-6 \mathrm{~b}\right), 3.51$ (ddd, $1 \mathrm{H}$, $\mathrm{H}-5), 3.38$ (d, $1 \mathrm{H}, \mathrm{H}-3), 1.45,1.39\left(2 \mathrm{~s}, 6 \mathrm{H}, \mathrm{CMe}_{2}\right) ;{ }^{13} \mathrm{C} \mathrm{NMR}\left(125.7 \mathrm{MHz}, \mathrm{CDCl}_{3}\right): \delta=$ 136.6-128.0 (Ph), $112.5\left(\mathrm{CMe}_{2}\right), 105.0(\mathrm{C}-2), 81.5(\mathrm{C}-4), 78.8(\mathrm{C}-3), 73.6,73.5\left(\mathrm{CH}_{2} \mathrm{Ph}\right)$, 71.6 (C-1), 61.0 (C-5), 60.4 (C-6), 27.1, $25.9\left(\mathrm{CMe}_{2}\right)$; FABMS: $\left.m / z 332(50 \% \text {, [M-Me] }]^{+}\right)$. Anal. Calcd for $\mathrm{C}_{17} \mathrm{H}_{21} \mathrm{~N}_{3} \mathrm{O}_{5}$ : C 58.78, H 6.09, N 12.09. Found: C, 58.84; H, 6.36; N, 11.99 .

5-Azido-5-deoxy-3,4-O-(o-xylylene)- $\alpha$-L-sorbopyranose (13). A solution of 12 (366 mg, $1.19 \mathrm{mmol})$ in 90\% aq TFA $(3.0 \mathrm{~mL})$ was stirred at room temperature for $30 \mathrm{~min}$. The mixture was concentrated and repeatedly co-distilled with water. The residue was purified by column chromatography (2:1 EtOAc-petroleum ether) to yield $\mathbf{1 3}$ (347 $\mathrm{mg}$, 95\%); $R_{f} 0.37$ (3:1 EtOAc-petroleum ether); $[\alpha]_{\mathrm{D}}=-229.7\left(c\right.$ 1.0, $\left.\mathrm{CH}_{2} \mathrm{Cl}_{2}\right)$; IR $(\mathrm{KBr}): v_{\max }$ $=3079,2940,2108,1653,1449,1377,1262,1094,764 \mathrm{~cm}^{-1} ;{ }^{1} \mathrm{H}$ NMR (500 MHz, $\left.\mathrm{CDCl}_{3}\right)$ : $\delta=7.29-7.12(\mathrm{~m}, 4 \mathrm{H}, \mathrm{Ph}), 5.12,5.06\left(2 \mathrm{~d}, 2 \mathrm{H},{ }^{2} J_{\mathrm{H}, \mathrm{H}}=14.0 \mathrm{~Hz}, \mathrm{CHPh}\right), 5.03,4.97(2 \mathrm{~d}, 2$ $\left.\mathrm{H},{ }^{2} J_{\mathrm{H}, \mathrm{H}}=14.5 \mathrm{~Hz}, \mathrm{CHPh}\right), 3.79\left(\mathrm{t}, 1 \mathrm{H}, J_{3,4}=J_{4,5}=9.0 \mathrm{~Hz}, \mathrm{H}-4\right), 3.65\left(\mathrm{dd}, 1 \mathrm{H}, J_{6 \mathrm{a}, 6 \mathrm{~b}}=\right.$ $\left.11.0 \mathrm{~Hz}, J_{5,6 \mathrm{a}}=7.0 \mathrm{~Hz}, \mathrm{H}-6 \mathrm{a}\right), 3.64$ (d, $\left.1 \mathrm{H}, J_{1 \mathrm{a}, 1 \mathrm{~b}}=11.0 \mathrm{~Hz}, \mathrm{H}-1 \mathrm{a}\right), 3.59$ (d, $\left.1 \mathrm{H}, \mathrm{H}-1 \mathrm{~b}\right)$, $3.60\left(\mathrm{dd}, 1 \mathrm{H}, J_{5,6 \mathrm{~b}}=3.5 \mathrm{~Hz}, \mathrm{H}-6 \mathrm{~b}\right), 3.49$ (m, $\left.1 \mathrm{H}, \mathrm{H}-5\right), 3.48$ (d, $\left.1 \mathrm{H}, \mathrm{H}-3\right) ;{ }^{13} \mathrm{C} \mathrm{NMR}$ 
$\left(125.7 \mathrm{MHz}, \mathrm{CDCl}_{3}\right): \delta=136.6-128.1(\mathrm{Ph}), 97.2(\mathrm{C}-2), 80.8(\mathrm{C}-4), 78.8(\mathrm{C}-3), 73.3$ $\left(\mathrm{CH}_{2} \mathrm{Ph}\right), 65.6(\mathrm{C}-1), 60.6(\mathrm{C}-5), 60.4(\mathrm{C}-6)$; FABMS: $m / z 330\left(50 \%,[\mathrm{M}+\mathrm{Na}]^{+}\right)$. Anal. Calcd for $\mathrm{C}_{14} \mathrm{H}_{17} \mathrm{~N}_{3} \mathrm{O}_{5}$ : C 54.72, H 5.58, N 13.67. Found: C 54.47, H 5.58, N 13.64.

(2R,3R,4R,5R)-2,5-Dihydroxymethyl-3,4-dihydroxypyrrolidine (DMDP, 1). To a solution of $11(400 \mathrm{mg}, 1.3 \mathrm{~mol})$ in $2: 1 \mathrm{MeOH}-\mathrm{H}_{2} \mathrm{O}(15 \mathrm{~mL})$ and aq $\mathrm{HCl}(37 \%, 1 \mathrm{~mL})$, $\mathrm{Pd} / \mathrm{C}(370 \mathrm{mg}$ ) was added and the resulting suspension was hydrogenated at 4 bars for $24 \mathrm{~h}$, then filtered through Celite and concentrated. The the crude residue was dissolved in water, neutralized by treatment with Amberlite IRA $68\left(\mathrm{OH}^{-}\right)$ion-exchange resin, and freezed dried to afford the fully unprotected 2 as an oily residue (202 $\mathrm{mg}$, 95\%), having spectroscopic properties identical to those reported in the literature. ${ }^{1}$

$(2 R, 3 R, 4 R, 5 S)-2,5-D i h y d r o x y m e t h y l-3,4-d i h y d r o x y p y r r o l i d i n e \quad$ (DGDP, 2). Catalytic hydrogenation of $\mathbf{1 3}$ (40 $\mathrm{mg}, 0.13 \mathrm{~mol}$ ), following the procedure above described for the preparation of $\mathbf{1}$, gave 3 (203 $\mathrm{mg}, 96 \%$ ) having spectroscopic properties identical to those reported in the literature. ${ }^{2}$

\section{References}

(1) (a) Welter, A.; Jadot, J.; Dardenne, G.; Marlier, M.; Casimir, J. Phytochemistry 1976, 15, 747. (b) Fechter, M. H.; Gradnig, G.; Berger, A.; Mirtl, C.; Schmid, W.; Stütz, A. E. Carbohydr. Res 1998, 309, 367.

(2) (a) Liu, K. K.-C.; Kajimoto, T.; Chen, L.; Ichikawa, Y.; Wong, C.-H. J. Org. Chem. 1991, 56, 6280. (b) Reitz, A. B.; Baxter, E. W. Tetrahedron Lett. 1990, 31, 6777. 

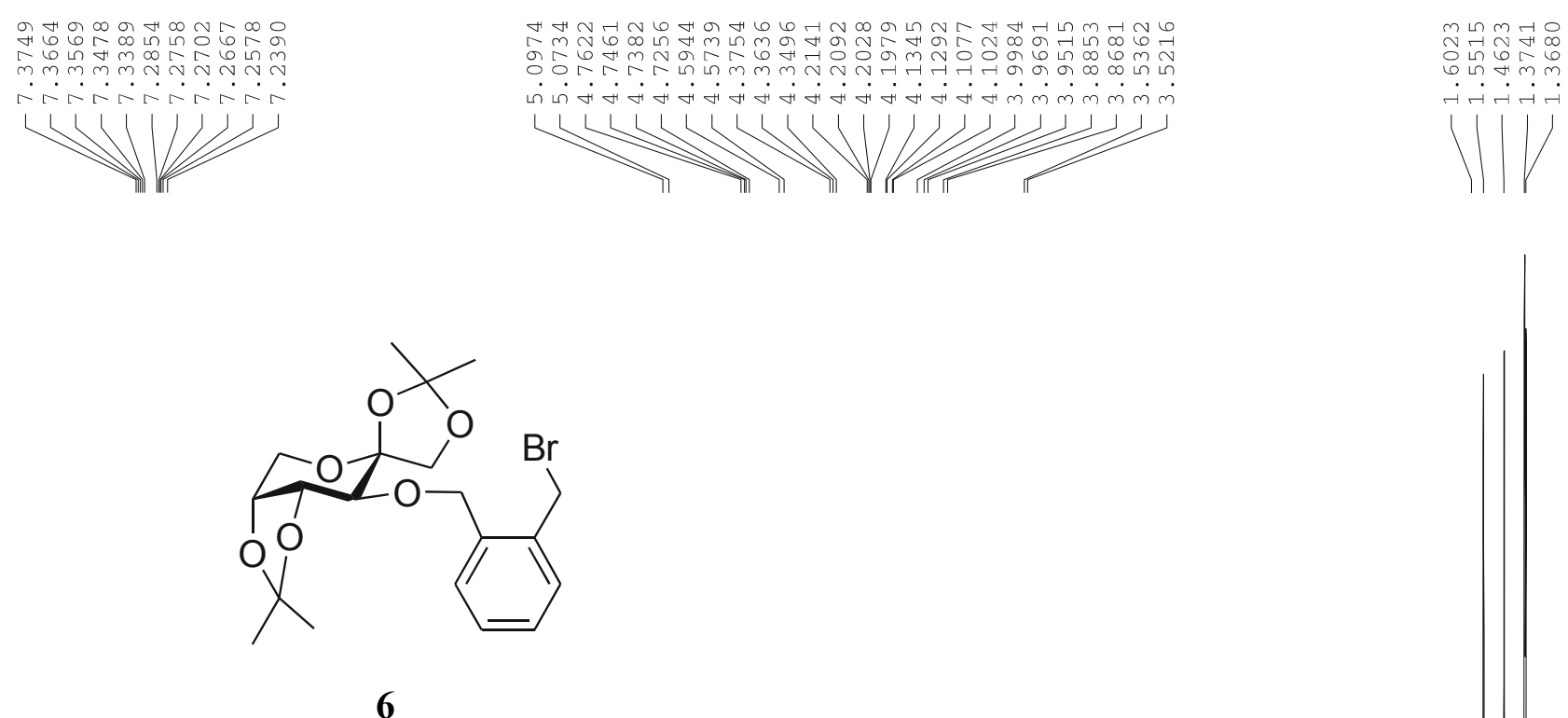

$\mathrm{CDCl}_{3}, 500 \mathrm{MHz}$

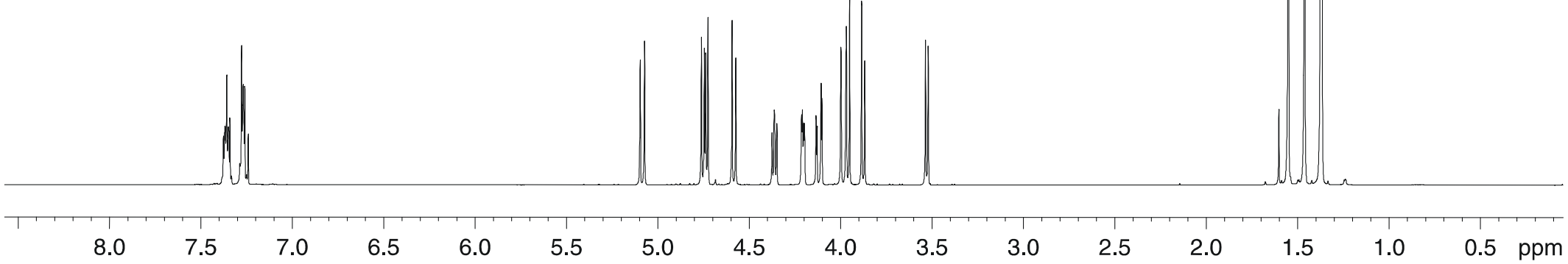



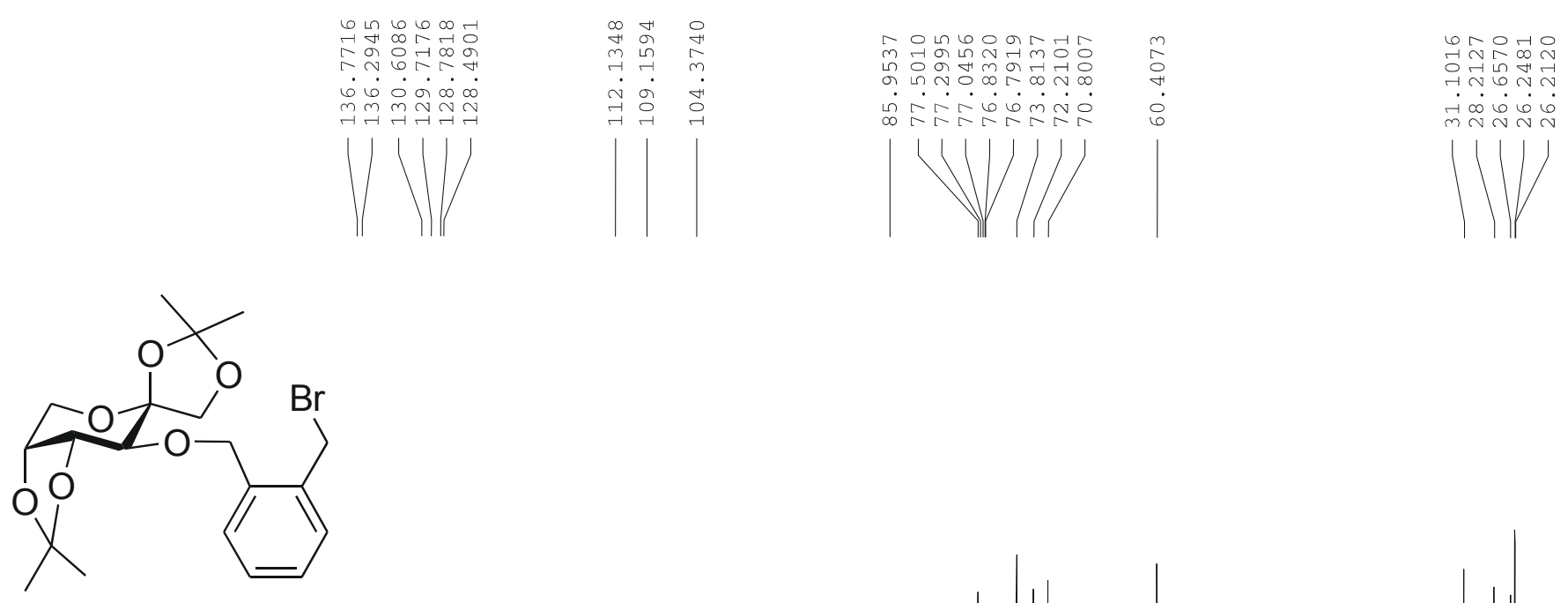

6

$\mathrm{CDCl}_{3}, 125.7 \mathrm{MHz}$

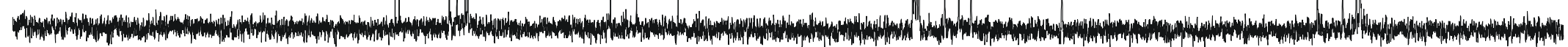

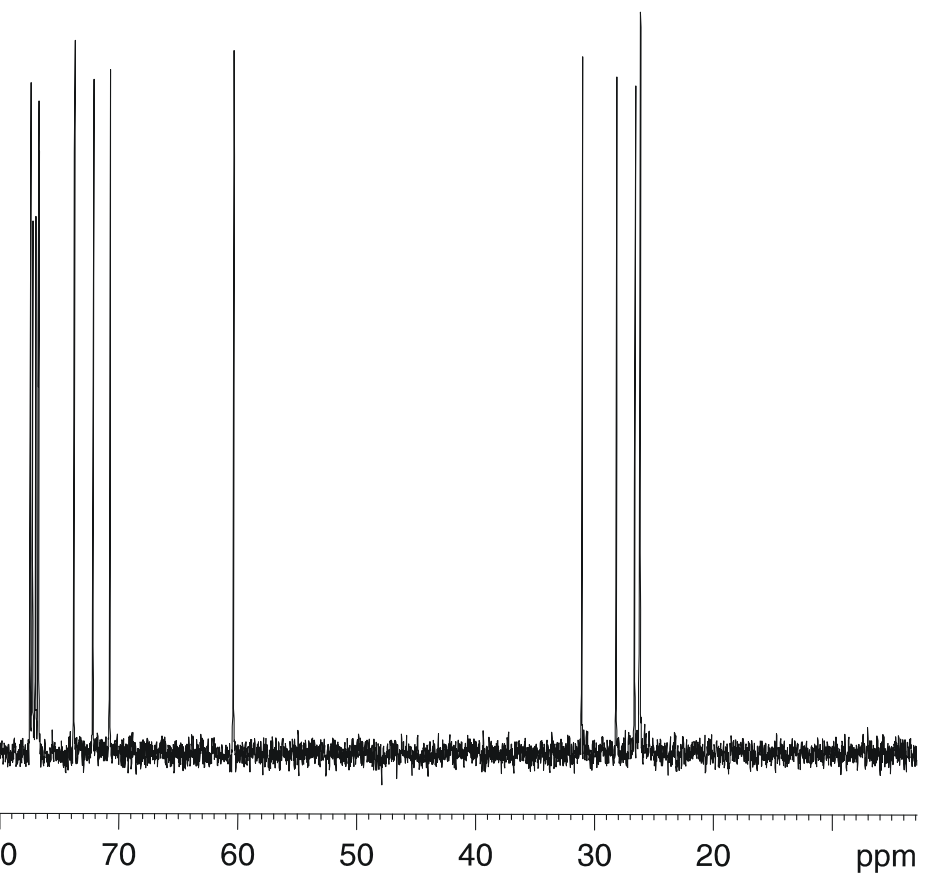

$\begin{array}{lllll}170 & 160 & 150 & 140 & 130\end{array}$

120

10100

$90 \quad 80$

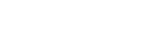

$50 \quad 40 \quad 30 \quad 20$

ppm 


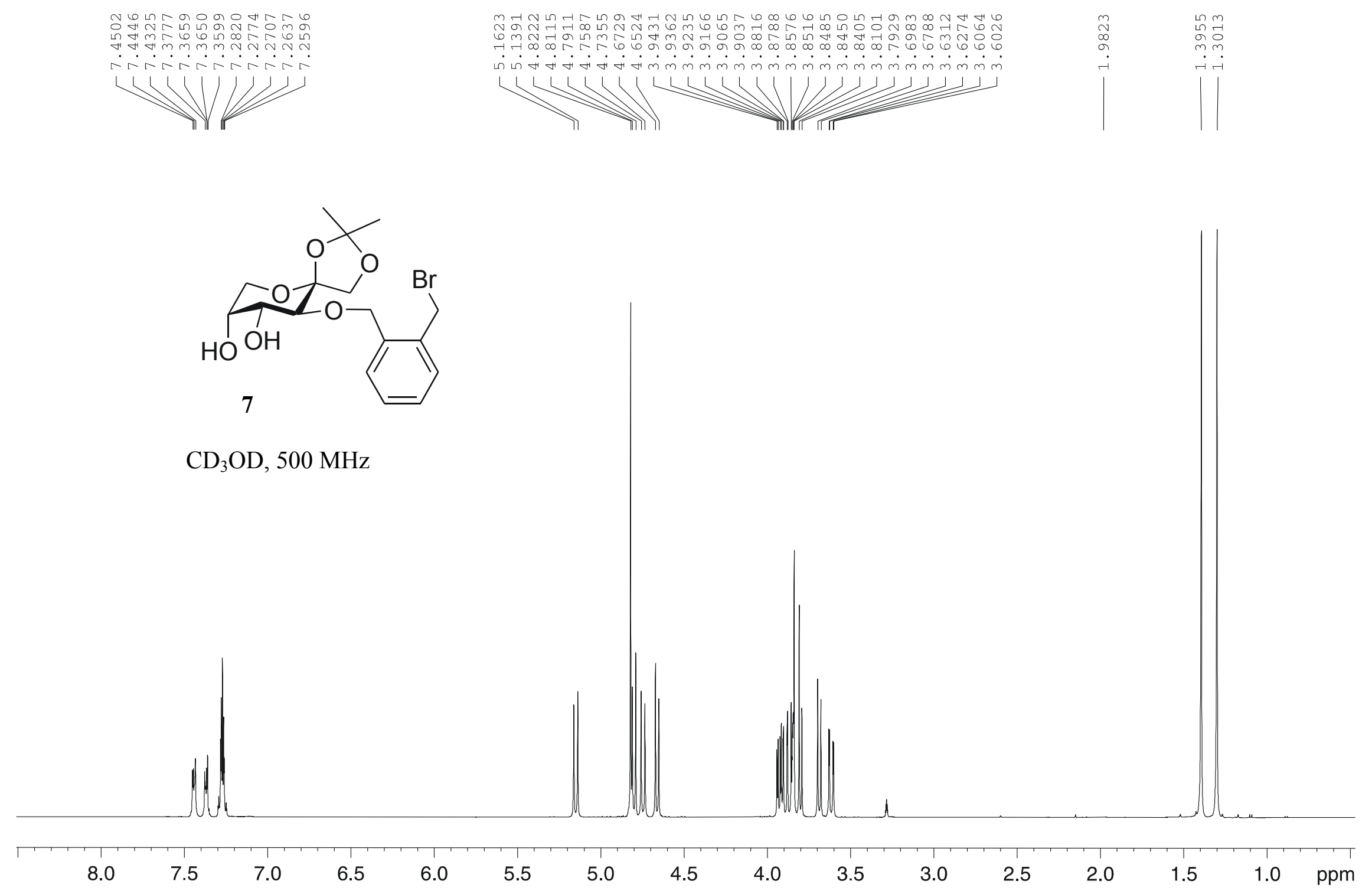



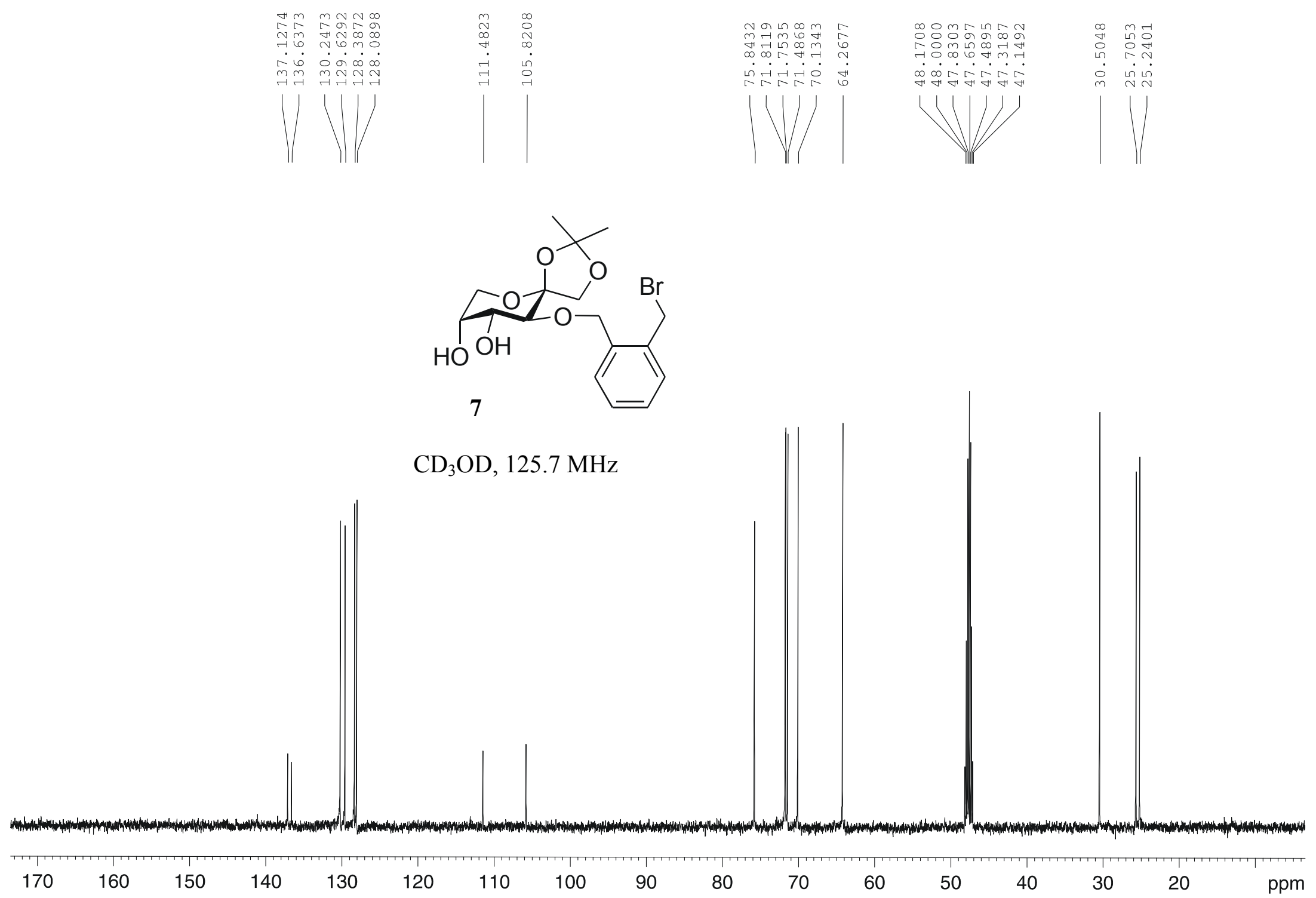


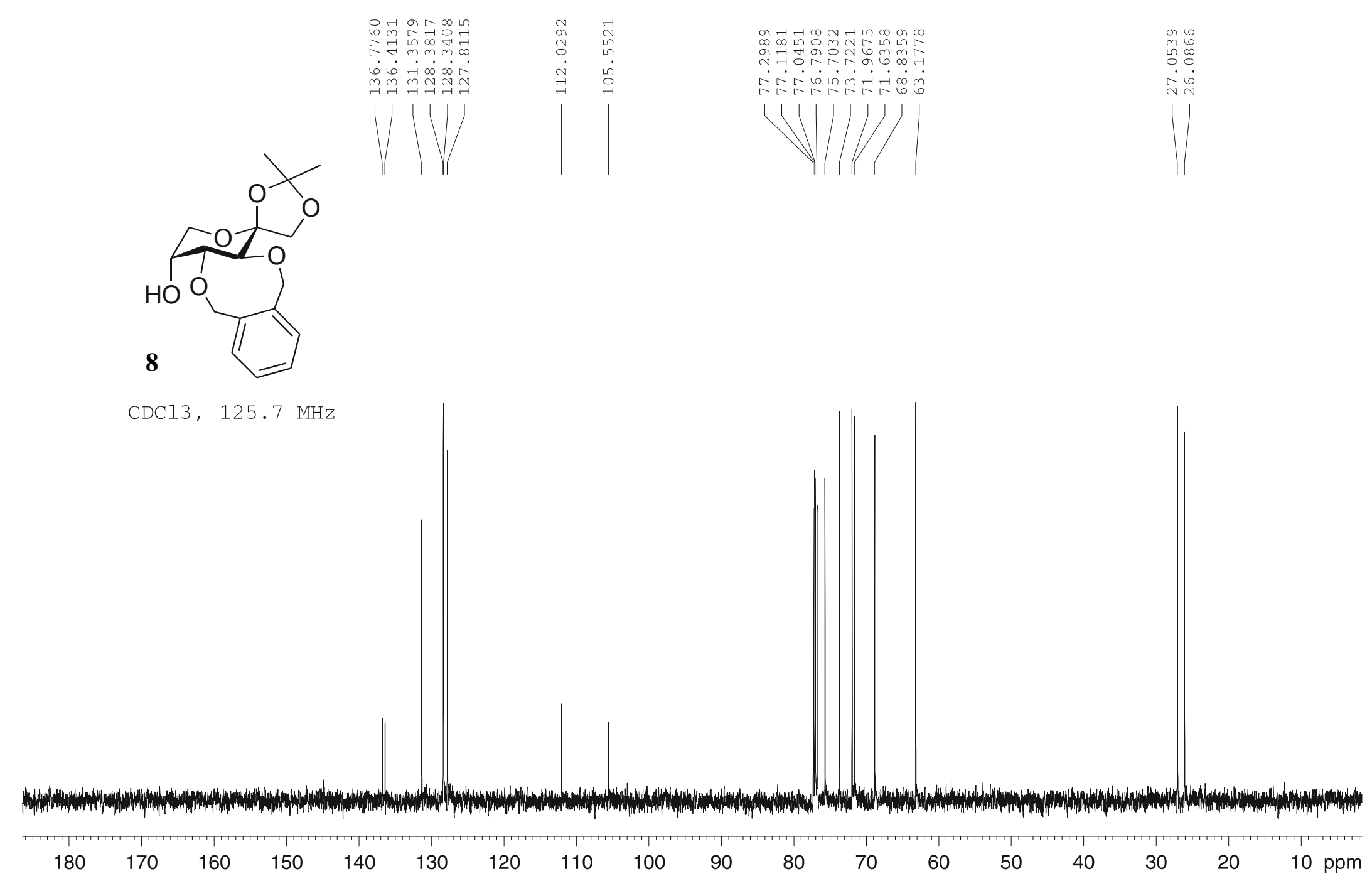



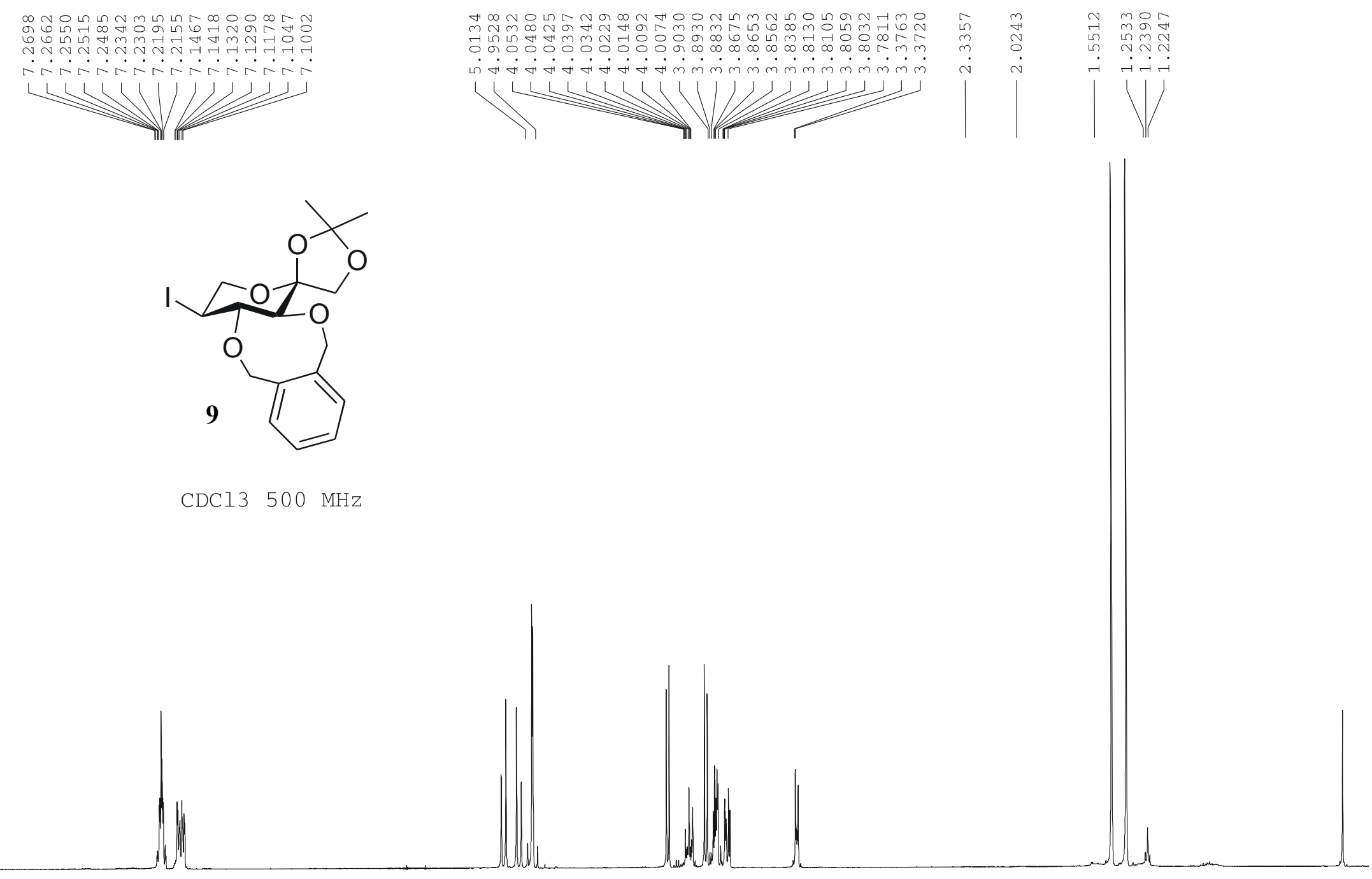

CDCl3 $500 \mathrm{MHz}$

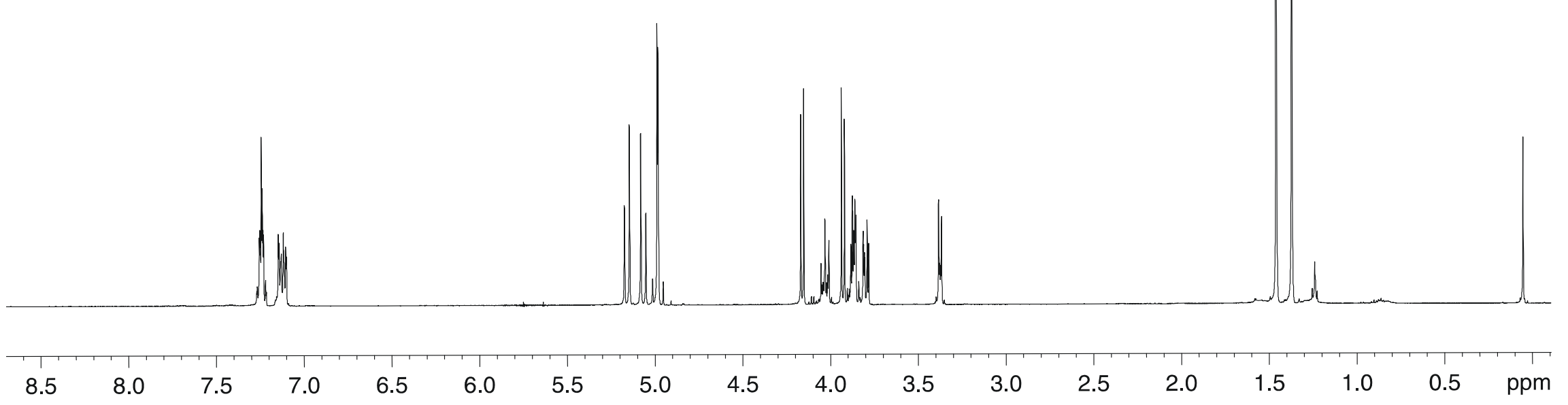



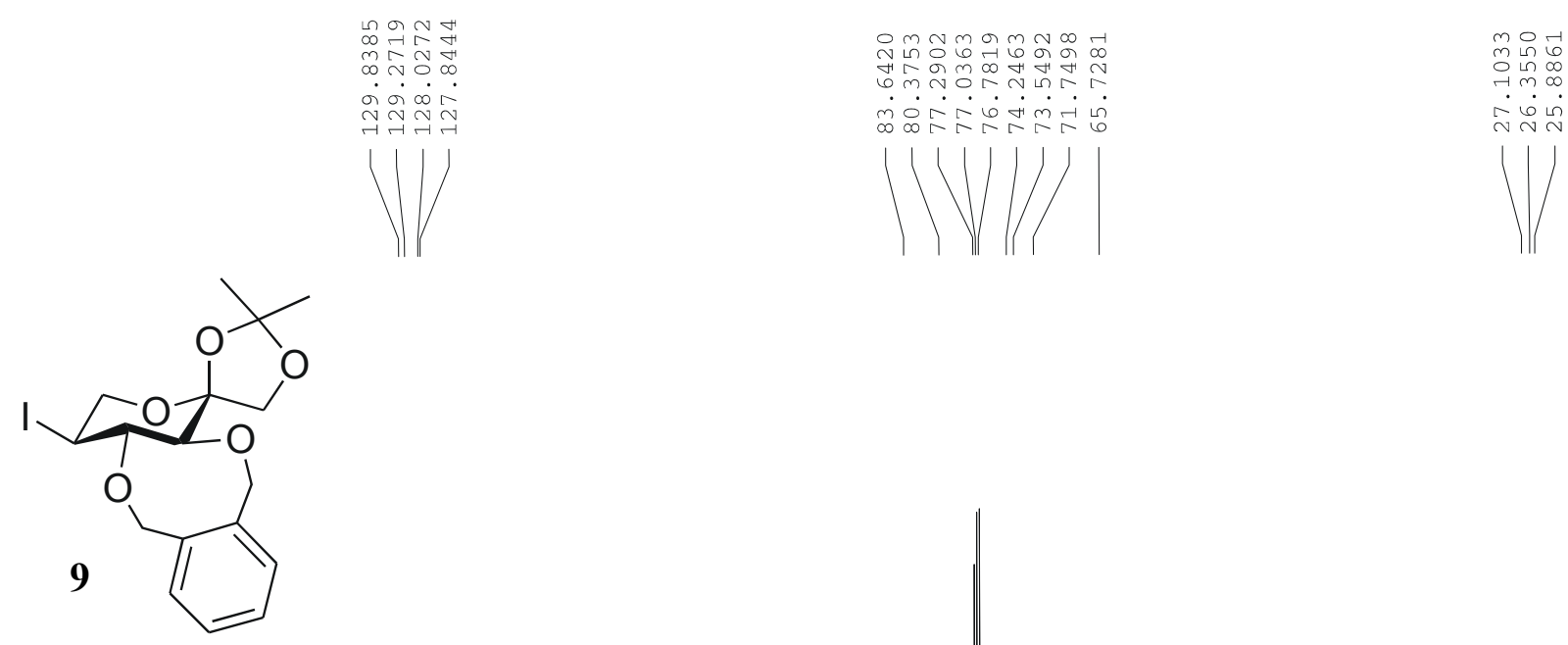

CDC13 125.7 MHz

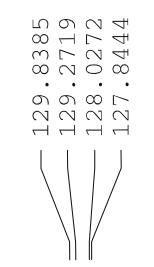

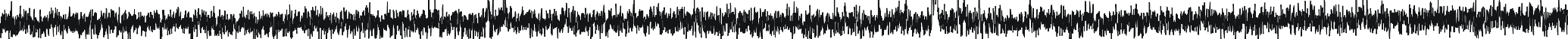



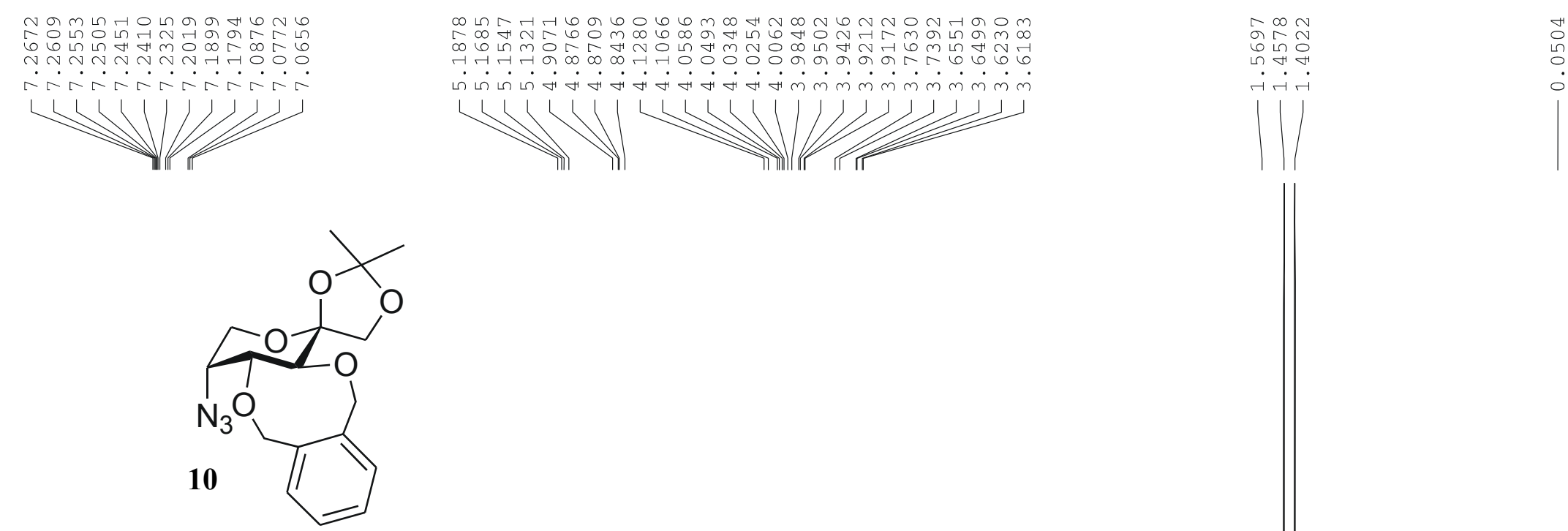

CDCl3 $400 \mathrm{MHz}$

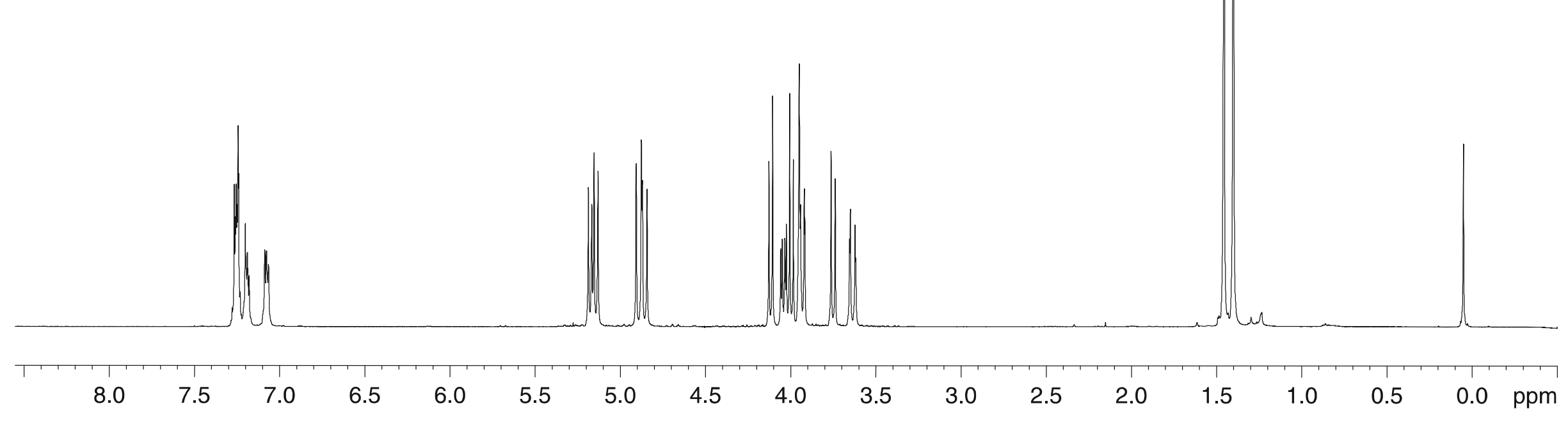



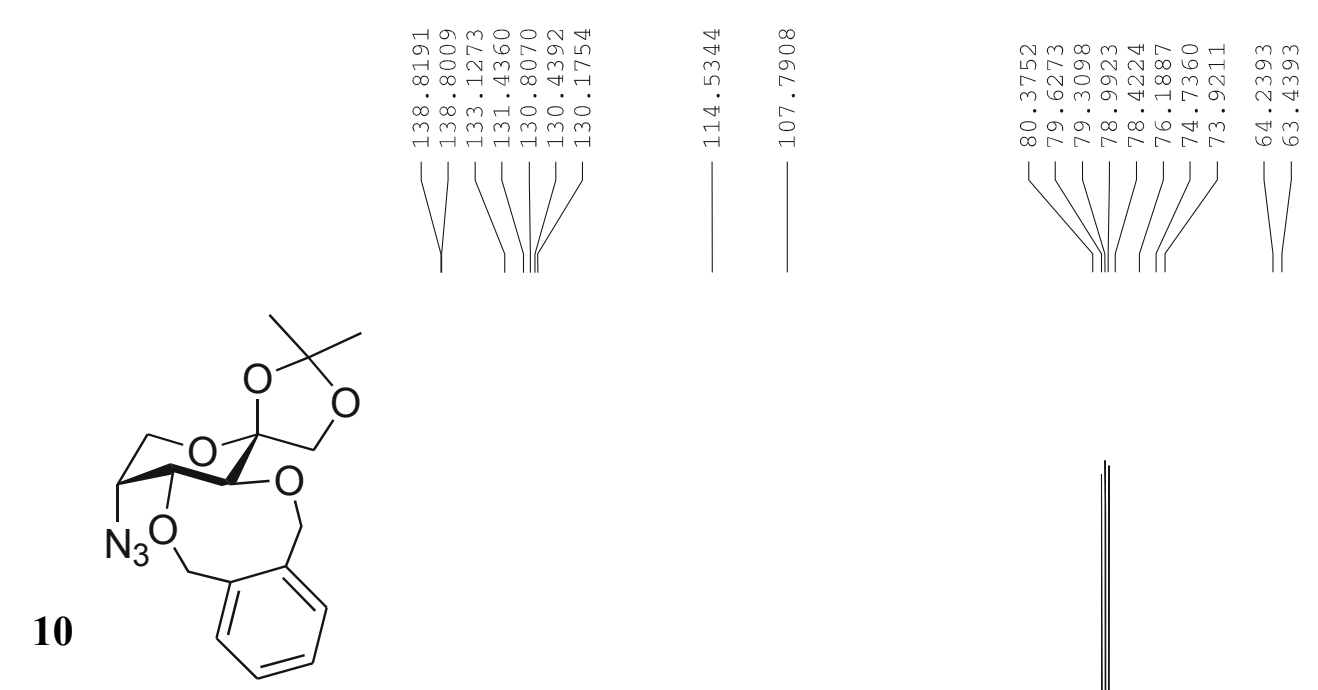

$\mathrm{CDCl}_{3}, 100.6 \mathrm{MHz}$

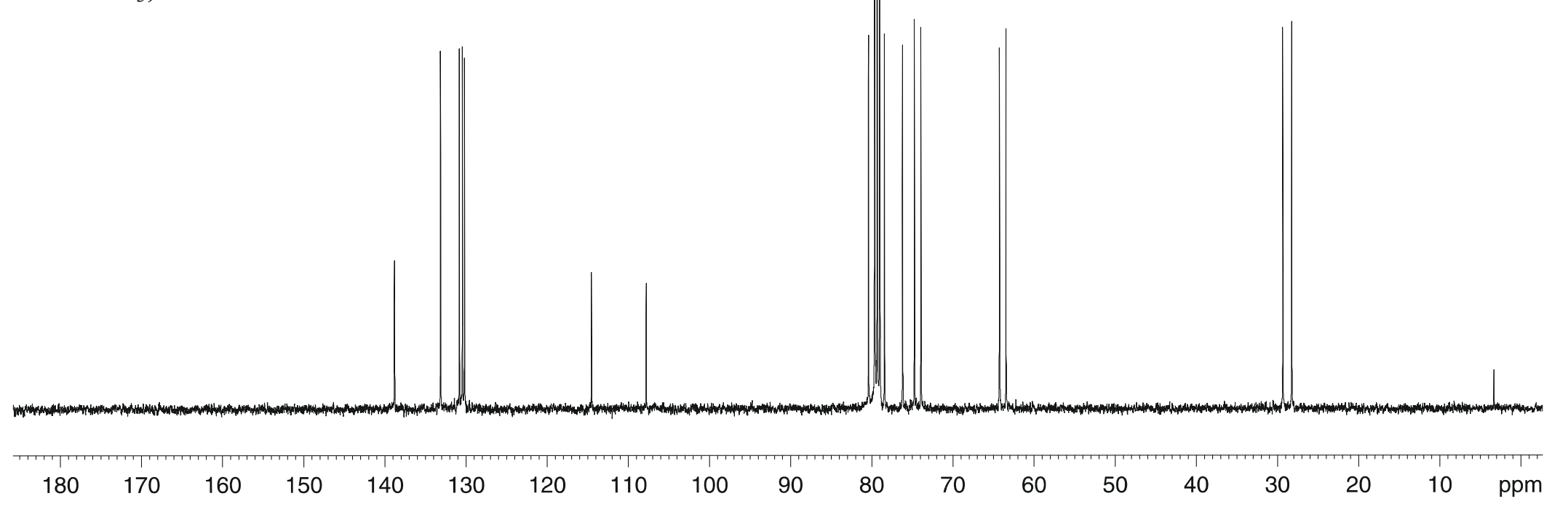



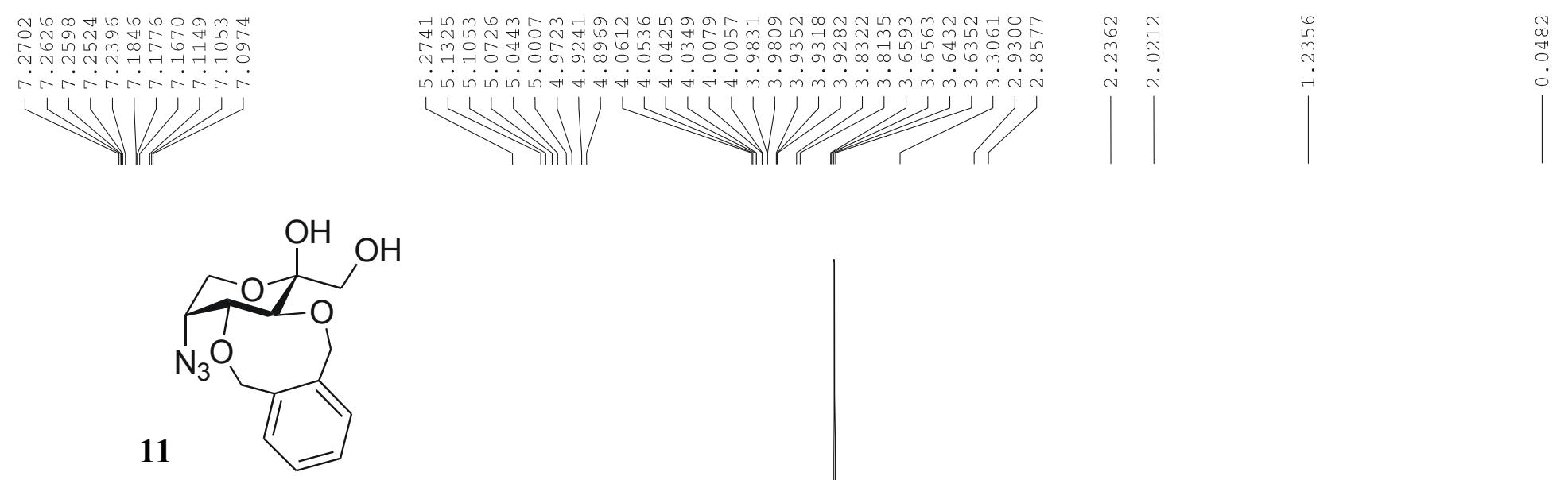

CDC13 $500 \mathrm{MHz}$

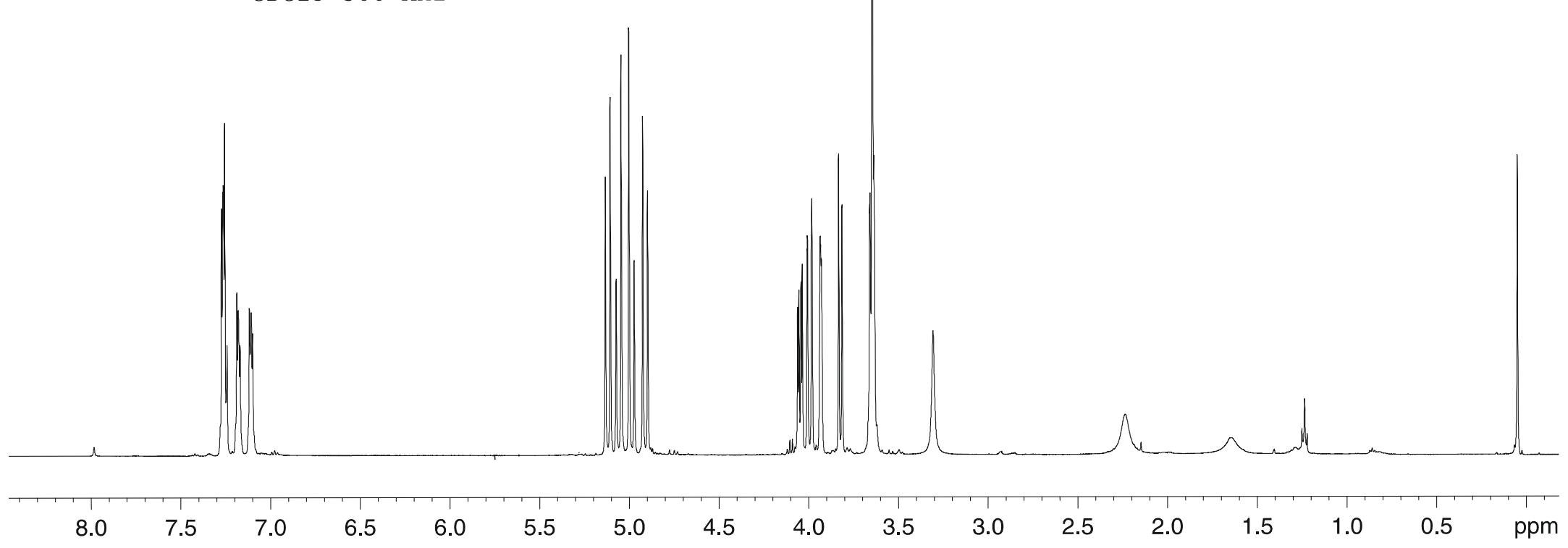



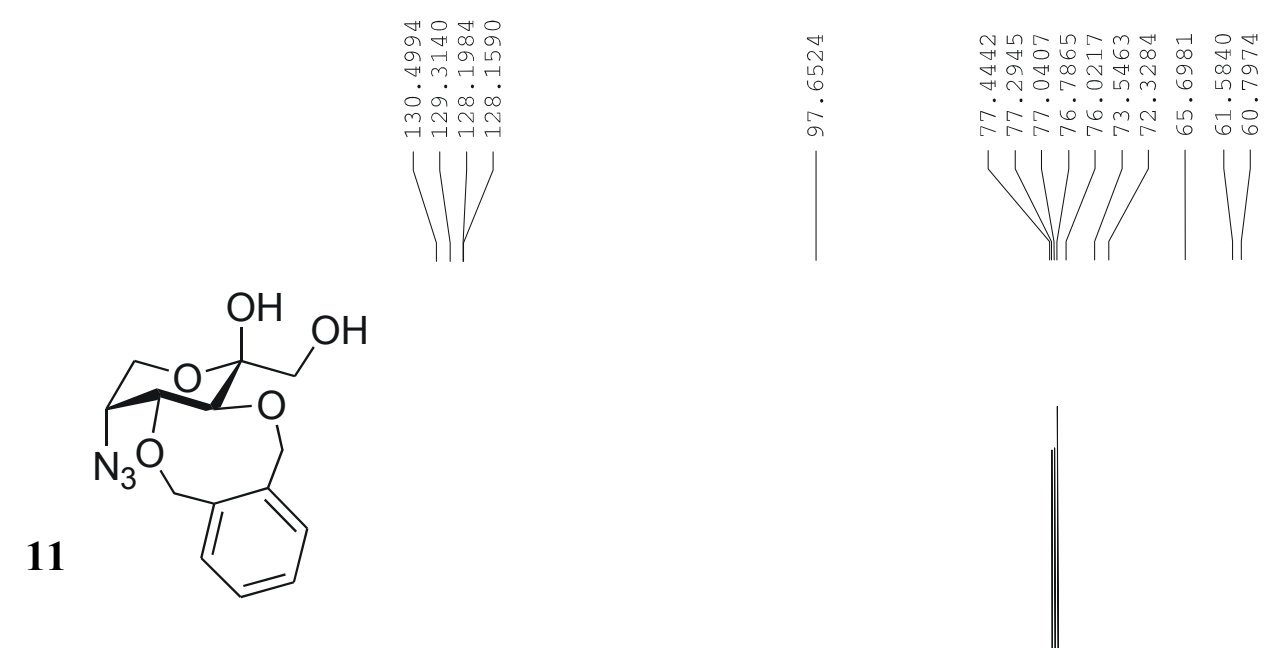

CDC13 125.7 MHz

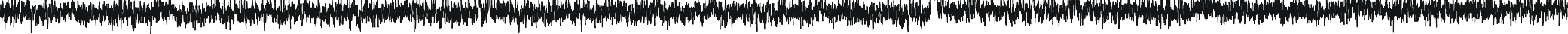



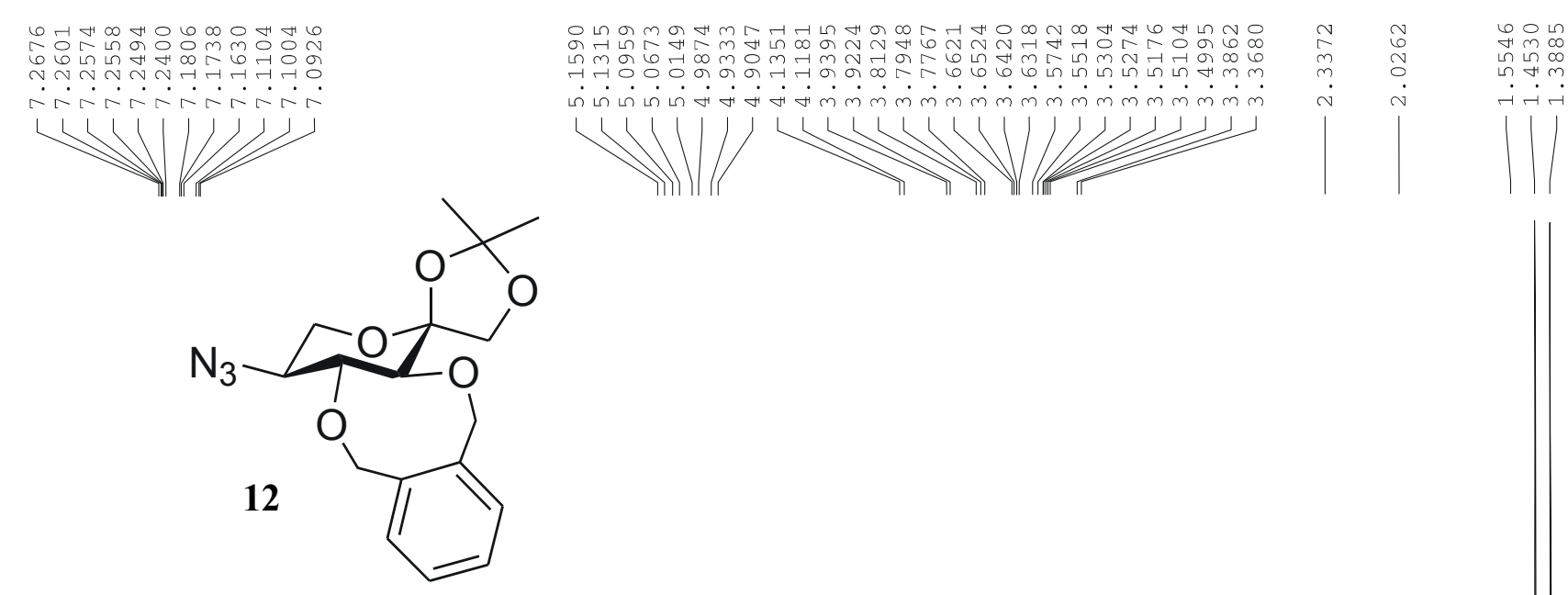

CDC13 $500 \mathrm{MHz}$

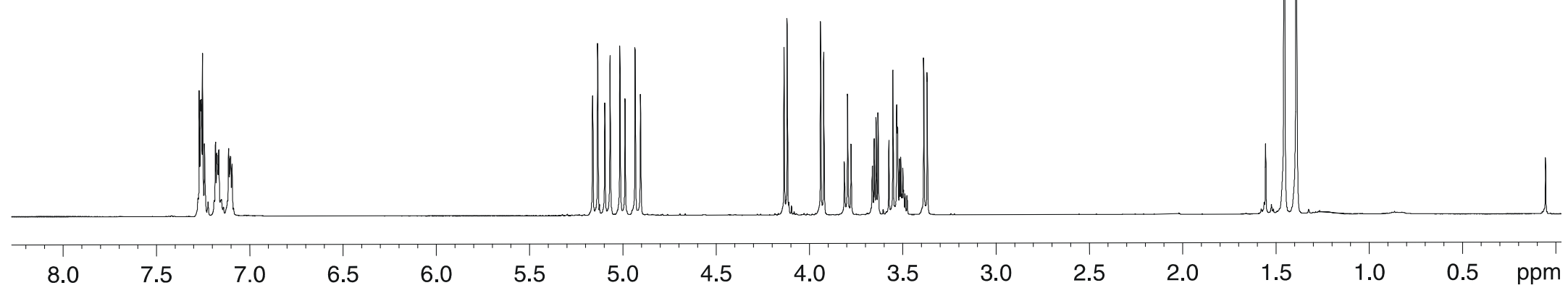



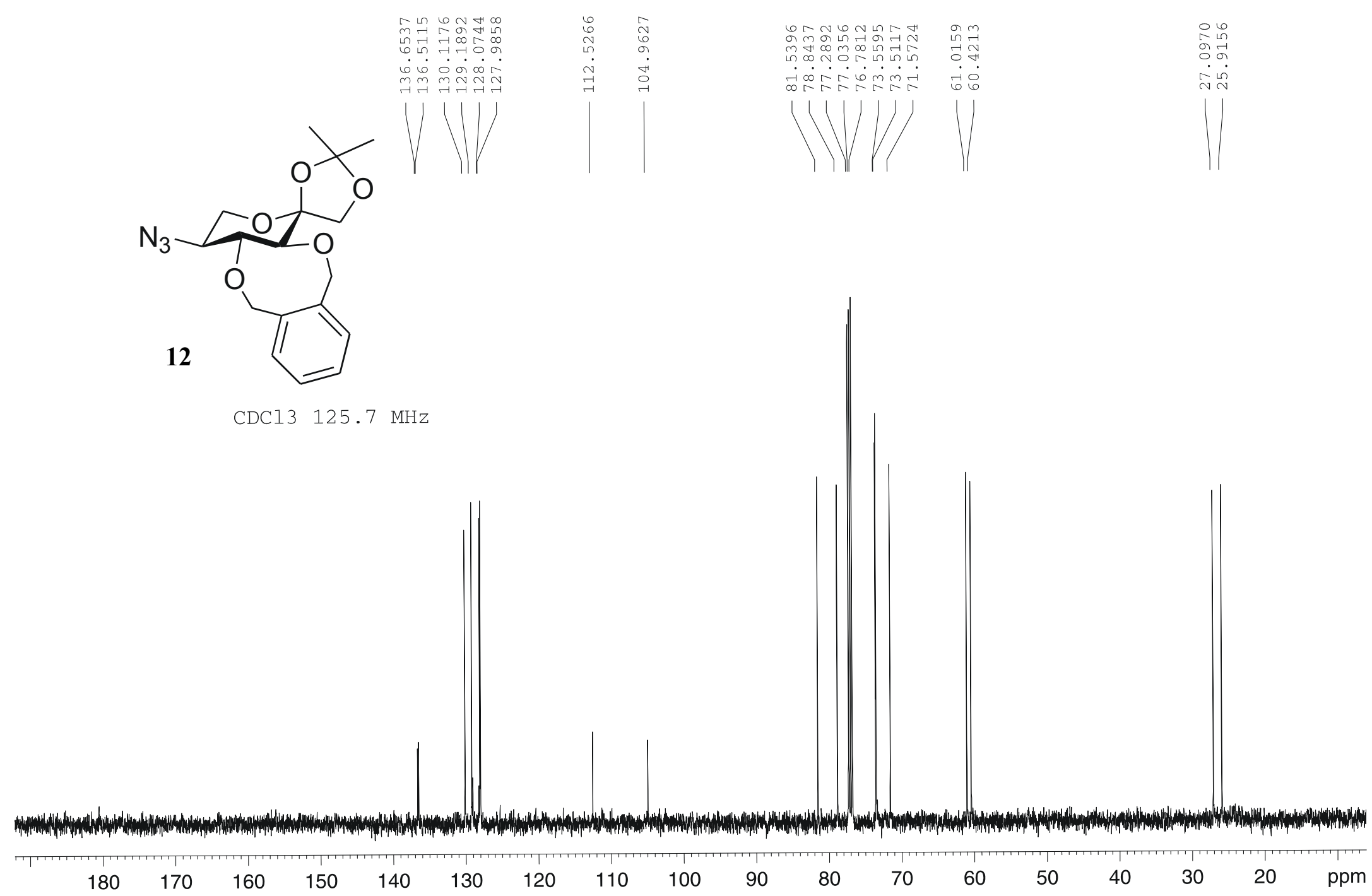


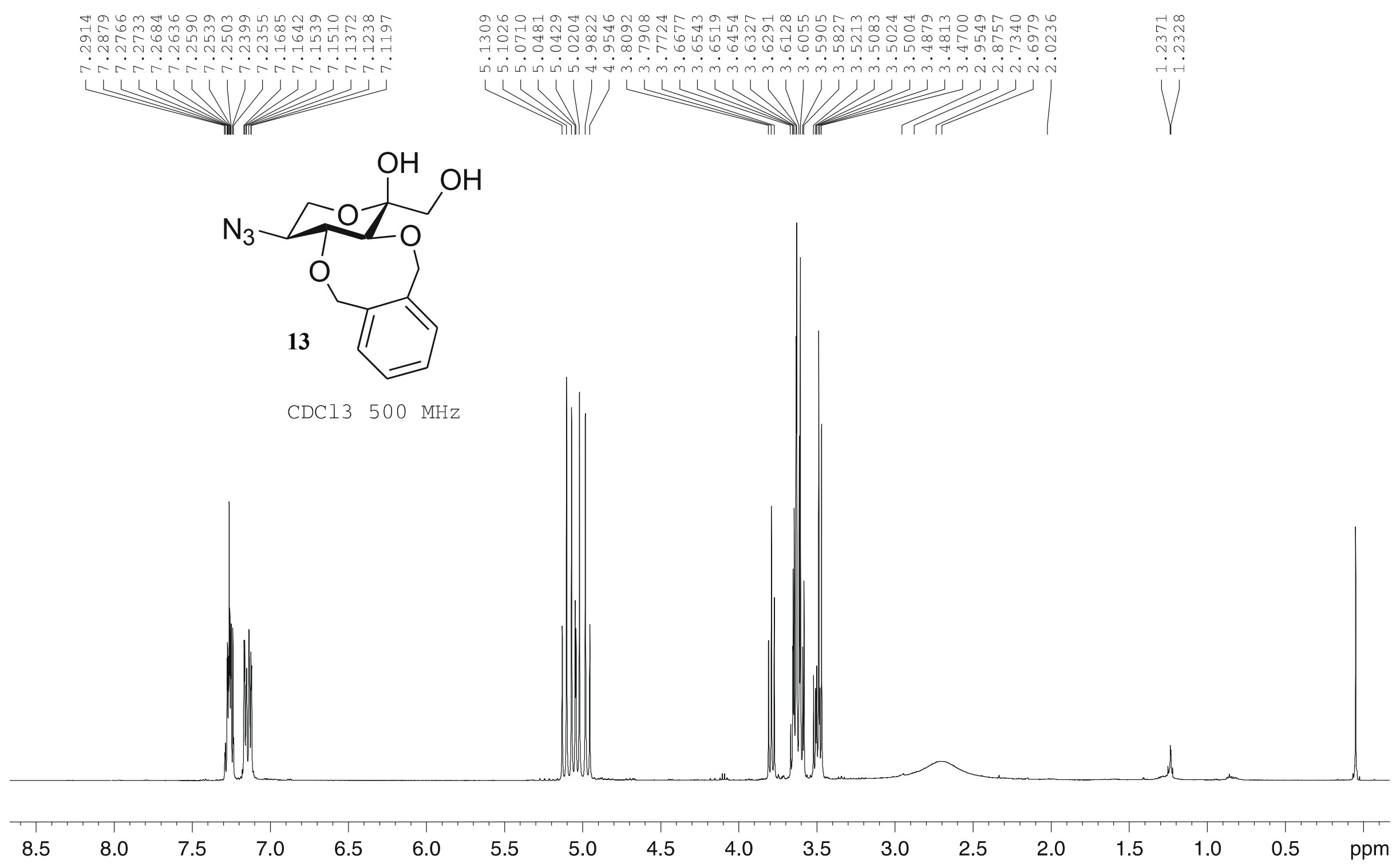



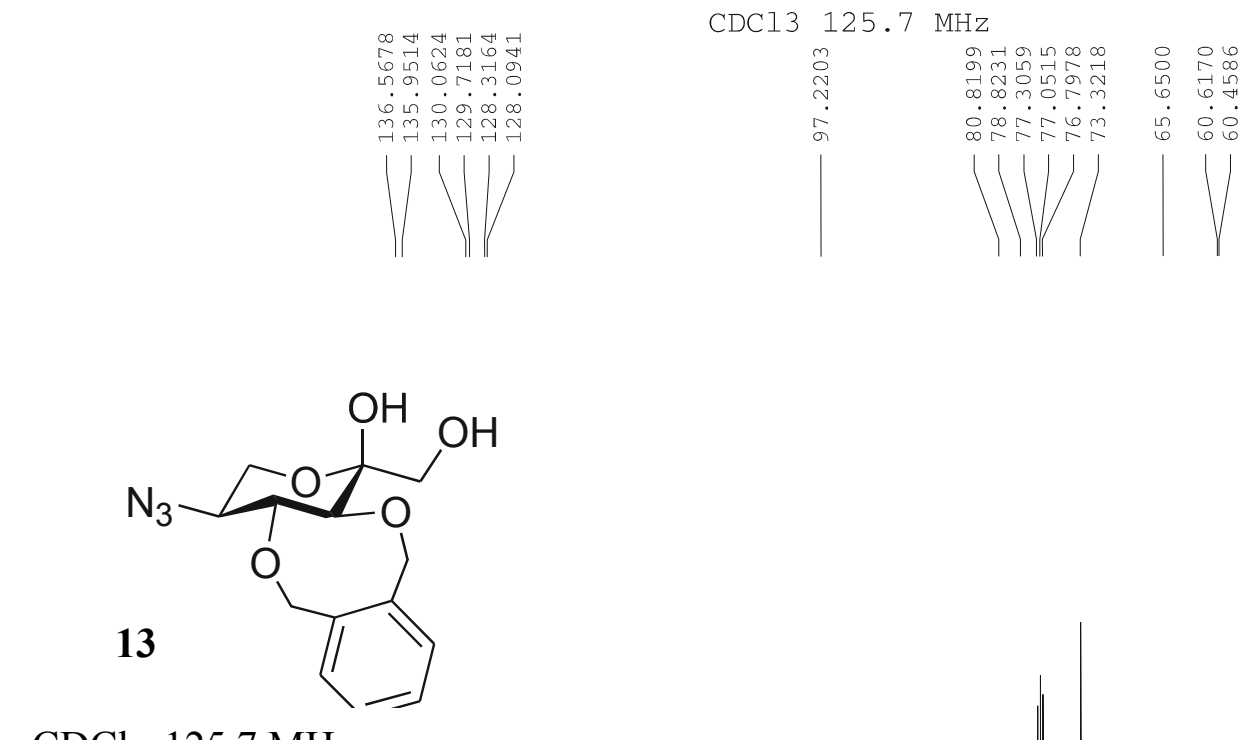

$\mathrm{CDCl}_{3}, 125.7 \mathrm{MHz}$

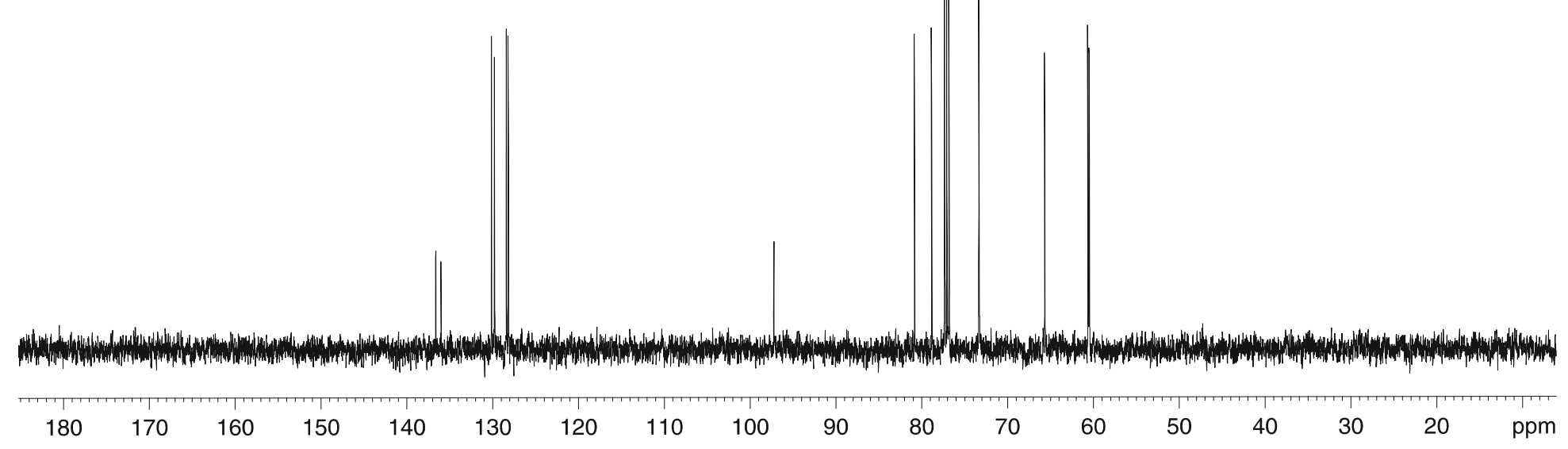

